\title{
Linearization strategies for the Iterative Nonlinear Contrast Source method for full-wave simulation of nonlinear ultrasound fields
}

\author{
Martin D. Verweij, Libertario Demi and Koen W.A. van Dongen \\ Laboratory of Acoustical Wavefield Imaging, Faculty op Applied Sciences, Delft University of \\ Technology, Lorentzweg 1,2628 CJ Delft,The Netherlands,m.d.verweij@tudelft.nl
}

\begin{abstract}
The Iterative Nonlinear Contrast Source (INCS) method is a full-wave method for the accurate computation of wide-angle, pulsed, nonlinear ultrasound fields appearing in, e.g., medical echoscopy. The method is based on the Westervelt equation and considers the occurring nonlinear term as a distributed contrast source that operates in a linear background medium. This formulation leads to an integral equation, which is solved in an iterative way. The original INCS method uses a Neumann scheme to successively approximate the nonlinear wave field in homogeneous, lossless, nonlinear media. To cope with attenuative and/or inhomogeneous nonlinear media, additional contrast sources may be introduced. Since these deteriorate the convergence rate of the Neumann scheme, more advanced iterative solution schemes like Bi-CGSTAB are required. To overcome the difficulty that such schemes only apply to linear integral equations, the nonlinear contrast source is linearized, at the cost of a significant systematic error in the fourth and higher harmonics. In this paper, a strategy is proposed in which the relevant iterative solution scheme is restarted with an updated version of the linearized contrast source. Results demonstrate the effectiveness of this strategy in eliminating the systematic error. In addition, it is shown that the same approach also improves the convergence rate in case of nonlinear propagation in media with attenuation.
\end{abstract}

Keywords: $43.25 . \mathrm{Cb}, 43.25 . \mathrm{Jh}, 43.80 . \mathrm{Qf}$

PACS: Ultrasound, nonlinear acoustics, integral equation, iterative solution scheme, linearization

\section{INTRODUCTION}

The accurate simulation of nonlinear acoustic wave fields is important for the development and assessment of medical ultrasound modalities that involve higher harmonics, such as harmonic imaging and HIFU. Various methods have been developed to perform these simulations, all having their specific properties [1]. One of these is the Iterative Nonlinear Contrast Source (INCS) method [2]. This full-wave method has particularly been developed for the accurate computation of the higher harmonics in pulsed diagnostic ultrasound beams that are steered over large angles. Originally, an iterative Neumann scheme was employed to find increasingly accurate approximations of the nonlinear wave field in homogeneous, lossless media. Recently, a linearized version of INCS [3] was introduced to allow for more advanced iterative solution schemes such as BiCGSTAB [4]. This approach yields an improved convergence rate, which is necessary when dealing with attenuative and/or inhomogeneous nonlinear media. However, this comes at the cost of a systematic error due to the linearization. In this paper we present an iteration strategy that leads to a reduction of this systematic error. The strategy will also be used to improve the convergence rate in case of nonlinear media with attenuation. 


\section{THE ORIGINAL INCS METHOD}

The original INCS method [2] is based on the lossless version of the Westervelt equation [5] for a homogeneous, nonlinear medium

$$
\nabla^{2} p(x, t)-\frac{1}{c_{0}^{2}} \frac{\partial p(x, t)}{\partial t^{2}}=-S_{\mathrm{pr}}(x, t)-\frac{\beta}{\rho_{0} c_{0}^{4}} \frac{\partial p^{2}(x, t)}{\partial t^{2}},
$$

where $p$ is the acoustic pressure, $\rho_{0}$ the mass density, $c_{0}$ the acoustic wave speed, $\beta$ the coefficient of nonlinearity, and $S_{\mathrm{pr}}$ the primary source representing the transducer. Considering weakly to moderately nonlinear situations, we give the nonlinear term in Eq. (1) the role of a nonlinear contrast source $S_{\mathrm{nl}}[p]$ that adds a relatively small correction $\tilde{p}$ to the linear field $p^{(0)}$. The latter is the field that would exist in the absence of nonlinearity. The fields $p^{(0)}$ and $\tilde{p}$ are given by

$$
p^{(0)}=G *_{x, t} S_{\mathrm{pr}}, \quad \tilde{p}=G *_{x, t} S_{\mathrm{nl}}[p] .
$$

Here, $G$ is the Green's function (spatiotemporal impulse response) of a linear 'background' medium with parameters $c_{0}$ and $\rho_{0}$, and $*_{x, t}$ denotes a four-dimensional convolution over space and time. Since $p=p^{(0)}+\tilde{p}$, we find that

$$
p=p^{(0)}+G *_{x, t} S_{\mathrm{nl}}[p],
$$

which is an integral equation. In the current case of a homogeneous and lossless nonlinear medium, the total pressure may be found by using the iterative Neumann scheme

$$
p^{(j)}=p^{(0)}+G *_{x, t} S_{\mathrm{nl}}\left[p^{(j-1)}\right], \quad j=1,2,3, \ldots
$$

The most involving task in the numerical evaluation of the scheme is the spatiotemporal convolution, which is efficiently performed with the FFT-based Filtered Convolution method [6].

\section{THE LINEARIZED INCS METHOD}

To deal with media that show spatially dependent attenuation, wave speed, or density of mass, the INCS method can easily be extended with additional contrast sources. For example, in media with inhomogeneous losses the nonlinear acoustic wave field is governed by [7]

$$
\nabla^{2} p-\frac{1}{c_{0}^{2}} \frac{\partial^{2} p}{\partial t^{2}}=-S_{\mathrm{pr}}-S_{\mathrm{nl}}[p]-S_{\mathrm{att}}[p],
$$

where the contrast source $S_{\text {att }}[p]$ can accommodate spatially dependent attenuation of any type, as well as the associated dispersion. These additional contrast sources may be stronger than the nonlinear contrast source $S_{\mathrm{nl}}[p]$ and may deteriorate the convergence rate of the Neumann scheme. The direct application of more advanced iterative solution schemes like Bi-CGSTAB is prohibited in the nonlinear context of the INCS method because these can only deal with linear integral equations. To overcome this difficulty, 

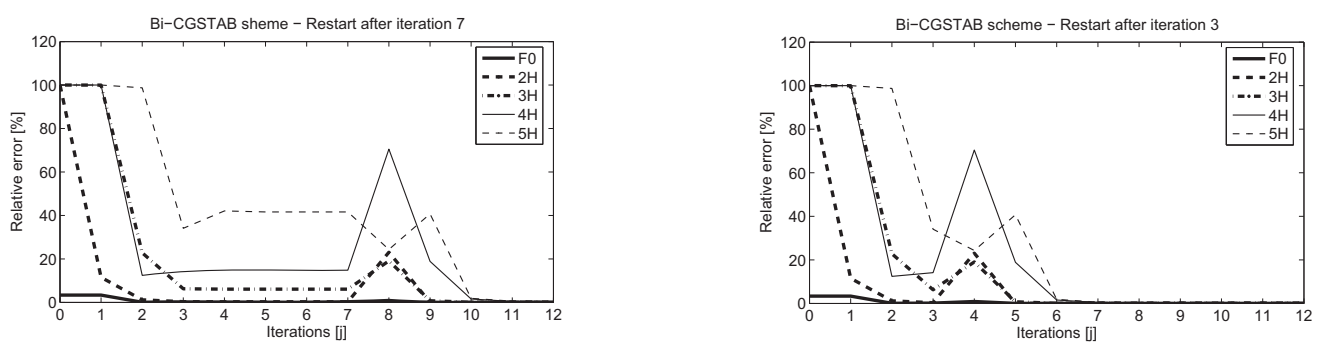

FIGURE 1. The relative error in the fundamental $(\mathrm{F} 0)$ and the second till the fifth harmonic $(2 \mathrm{H}-5 \mathrm{H})$ of a plane wave propagating in water. Left: relative errors for a restart after iteration $j=7$. Right: relative errors for a restart after iteration $j=3$. Relative errors have been determined with respect to the result of the Burgers equation.

it has recently been proposed to linearize the nonlinear contrast source by using the approximation $p^{2} \approx\left(p^{(0)}\right)^{2}+2 p^{(0)} \tilde{p}$, i.e. by assuming that the square of the correction $\tilde{p}$ may be neglected [3]. This idea may even be applied to the original INCS method by replacing $S_{\mathrm{nl}}[p]$ in Eq. (3) by

$$
S_{\mathrm{nl}}^{\text {new }}[p]=\frac{\beta}{\rho_{0} c_{0}^{4}} \frac{\partial}{\partial t^{2}}\left[-\left(p^{(0)}\right)^{2}+2 p^{(0)} p\right] .
$$

The linearization approach has been tested in combination with the Bi-CGSTAB scheme [4], both in cases without and with attenuation. In both cases the convergence rate improves over the non-linearized INCS method with a Neumann scheme, but linearization turns out to introduce significant systematic errors in the fourth and higher harmonics.

\section{ADVANCED ITERATION STRATEGIES FOR THE LINEARIZED INCS METHOD}

In this paper it is investigated whether the systematic error due to linearization can be reduced by a 'restart' strategy:

- Perform the first $J$ iterations with the linearization $p^{2} \approx\left(p^{(0)}\right)^{2}+2 p^{(0)} \tilde{p}$.

- Perform subsequent iterations with the linearization $p^{2} \approx\left(p^{(J)}\right)^{2}+2 p^{(J)} \tilde{p}$.

The performance of this restart strategy is demonstrated for a plane source ( $1 \mathrm{MHz}$ sine with three-cycle Gaussian envelope, maximum surface pressure $P_{0}=1 \mathrm{MPa}$ ) in water $\left(\rho_{0}=998 \mathrm{~kg} / \mathrm{m}^{3}, c_{0}=1482 \mathrm{~m} / \mathrm{s}, \beta=3.52\right)$. The Bi-CGSTAB scheme is employed. Figure 1 shows the relative error in the fundamental and the second till the fifth harmonic of the generated plane wave, at a distance $z=100 \mathrm{~mm}$ from the source. The errors in the left part apply to a restart of the iterative scheme after iteration $j=7$. For $j=4$ to 7 , the errors do not change, indicating that the initial iterative scheme has reached convergence at $j=4$. At this stage, the remaining errors are the systematic errors introduced by the linearization around $p^{(0)}$. After restart, the scheme reaches convergence again at $j=11$, at which time it turns out that the systematic errors in the higher harmonics have effectively been eliminated by the linearization around $p^{(7)}$. Of course, the same final 

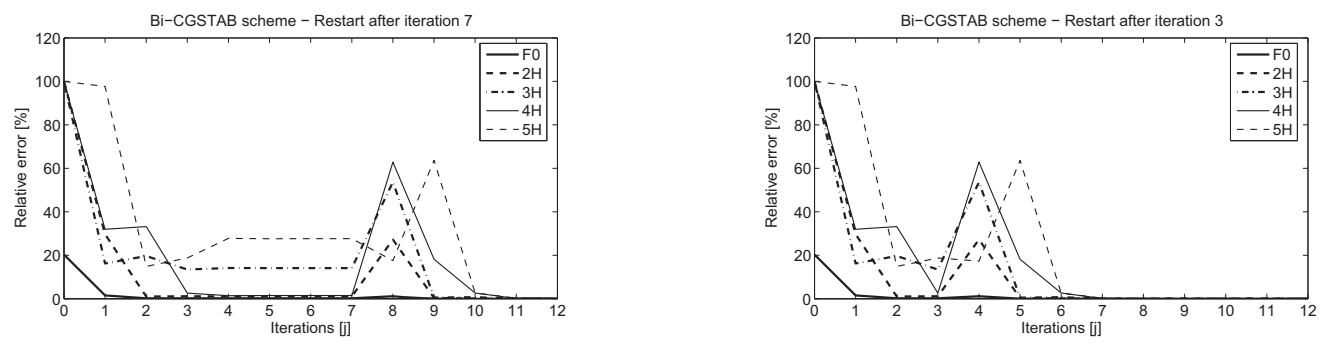

FIGURE 2. The relative error in the fundamental (F0) and the second till the fifth harmonic $(2 \mathrm{H}-5 \mathrm{H})$ of a plane wave propagating in a medium with frequency power law attenuation. Left: relative errors for a restart after iteration $j=7$. Right: relative errors for a restart after iteration $j=3$. Relative errors have been determined with respect to the result of the fully converged (15 iterations) non-linearized INCS method.

result may be obtained by stopping the initial scheme earlier, i.e. when the errors have almost settled. This fact is demonstrated in the right part of Fig. 1, in which a restart after $j=3$ leads to convergence at $j=7$, without observable systematic errors.

Further it is investigated whether the restart strategy may help to remedy the following effect: the convergence of the nonlinear INCS method for an attenuative, nonlinear medium is worse than for a lossless medium with the same nonlinearity, or for a linear medium with the same attenuation. Since we want to use the Bi-CGSTAB scheme, the restart strategy will again be combined with the linearization approach, as described above. To test the performance, a configuration with the same parameters as before is applied, but the medium now has a power frequency power law attenuation coefficient $\alpha=0.016\left|f / f_{0}\right|^{1.21} \mathrm{~Np} / \mathrm{cm}$, where $f_{0}=1 \mathrm{MHz}$. Figure 2 shows the relative error in the fundamental and the second till the fifth harmonic of a the propagating plane wave, at a distance $z=100 \mathrm{~mm}$ from the source. The same observations can be made as for the previous case. Upon comparing the right hand part of Fig. 2 with the right hand part of Fig. 1, the restarted scheme reaches convergence at the same number of iterations as the case without attenuation. When after restart convergence has been reached, the systematic errors caused by the linearization have virtually disappeared as well.

To conclude, the restart strategy is an efficient way to extend the applicability of the linearized INCS method to cases involving higher harmonics.

\section{REFERENCES}

1. M. D. Verweij and J. Huijssen, "Computational methods for nonlinear acoustic wavefields in homogeneous media," in Computational methods in nonlinear acoustics: Current trends, edited by C. Vanhille and C. Campos-Pozuelo, Research Signpost, Kerala, India, 2011, pp. 1-19.

2. J. Huijssen and M. D.Verweij, J. Acoust. Soc. Am. 127, pp. 33-44 (2010).

3. L. Demi, M. D. Verweij, N. de Jong, and K. W. A. vand Dongen, "Modeling nonlinear medical ultrasound via a linearized contrast source method," in 2010 IEEE International Ultrasonics Symposium Proceedings, San Diego, 2010, pp. 2175-2178.

4. H. A. van der Vorst, SIAM. J. Sci. Stat. Comput. 13, pp. 631-644 (1992).

5. M. F. Hamilton and C. L. Morfey, "Model equations," in Nonlinear acoustics, edited by M. F. Hamilton and D. T. Blackstock, Academic Press, San Diego, 1998, pp. 41-63.

6. M. D. Verweij and J. Huijssen, J. Acoust. Soc. Am. 125, pp. 1868-1878 (2009).

7. L. Demi, K. W. A. van Dongen, and M. D. Verweij, J. Acoust. Soc. Am. 129, pp. 1221-1230 (2011). 